\title{
Anomalous Bulk Viscosity of Two-Phase Fluids and Implications for Planetary Interiors
}

\author{
D. J. STEVENSON
}

\author{
Division of Geological and Planetary Sciences, California Institute of \\ Technology, Pasadena, California 91125
}

\begin{abstract}
A calculation is presented for the irreversible entropy production that accompanies the imposition of a pressure perturbation on a two-phase medium consisting of a dilute suspension of one phase (as droplets or snowflakes) in another (liquid) phase of significantly different composition. No metastability is allowed, and the relaxation process is then dominated by the finite diffusivity of solute. The fluid medium behaves as though it has a very large bulk viscosity (typical value $\sim 10^{12} \mathrm{P}$ in the lowfrequency limit). The minimum quality factor $Q$ for acoustic or tidal pressure oscillations is found to be typically $\sim 10^{2}-10^{3}$ and occurs at a frequency $\omega_{o} \sim 4 \pi D \eta \bar{s}$, where $D$ is the solute diffusivity and $\eta$ is the number density of suspended inclusions of average radius $\bar{s}$. For plausible parameter values, $\omega_{o}$ is in the range of planetary interest (e.g. $10^{-4} \mathrm{~Hz}$ ). At $\omega \leqslant \omega_{o}, Q \propto \omega^{-1}$; at $\omega_{0} \leq \omega \leq D \widehat{S}^{-2}, Q \propto \omega$; and at $\omega \geq D \bar{S}^{-2}, Q \propto \omega^{1 / 2}$. The model is applied to helium rain clouds in the deep interiors of giant planets and is found to be capable in principle of providing a tidal $Q-10^{5}$, needed to explain the volcanism of Io and resurfacing of Enceladus. The model is also applied to the earth's outer core and found to be marginally capable of explaining the attenuation of radial modes (notably ${ }_{o} S_{o}$ ) and potentially capable of providing significant attenuation of earth tides. However, quantification and application of the model are difficult because of large uncertainties in the nature of the required two-phase suspensions.
\end{abstract}

\section{INTRODUCTION}

When a pressure pulse passes through a material, the thermodynamic and structural responses are not instantaneous. A variety of relaxation processes ranging from microscopic (time scales as short as $10^{-12} \mathrm{~s}$ ) to macroscopic (arbitrarily long times) can cause a lag in the response of the system and an inevitable irreversible entropy production. This dissipation can be mathematically represented in terms of an 'anomalous' bulk viscosity and is an extensively studied phenomenon (see Landau and Lifshitz [1959, p. $304 \mathrm{ff}]$ for an elementary theoretical description and Litovitz and Davis, [1965] for a phenomenological and experimental review).

If the medium consists of two or more coexisting phases, then a particularly important relaxation process involves the delayed transformation of material from one phase to another as the pressure is varied. Multiphase media are common: large fractions of the earth's atmosphere are two-phase at any instant (i.e., contain both water vapor and condensed water), exsolution of gases causes many nearsurface geothermal hot waters and magmas to be bubbly and exhibit greatly modified acoustical characteristics [Kieffer, 1977], and we argue below that the fluid cores of planets are expected to have regions of saturation in which droplets or snowflakes of one phase are suspended in the remaining fluid. It is this latter situation that motivates the theory presented here. Vaisnys [1968] considered the attenuation of acoustic waves propagating through a system undergoing phase transition but considered only the effect of a finite reaction rate in his formulation rather than the unavoidable (and more readily quantifiable) irreversible

Copyright 1983 by the American Geophysical Union

Paper number 2B1617.

0148-0227/83/002B-1617\$05.00 entropy production arising from the transient diffusive profiles of heat and matter near inclusions (droplets or snowflakes) of the less abundant phase. In this paper, the reaction rate is assumed to be infinite (i.e., no kinetic inhibition or metastability is allowed), but the consequences of finite diffusivity are evaluated. Since the relaxation time of diffusive phenomena is frequency dependent, the present analysis differs fundamentally from that of Vaisnys, which assumes a frequency-independent relaxation. Unlike the problem of bubble-rich fluids, coexisting phases deep within planetary interiors have similar densities and compressibilities, and as a consequence, the theory presented here has little in common with the volcanological situation. The model considered is a dilute suspension of one phase in another of comparable density, subjected to an oscillating pressure field, assuming exact thermodynamic equilibrium at the phase boundaries at all times. Remarkably, this most basic model has not been completely analyzed before. However, Loper and Fearn [1982] have independently developed a very closely related and complementary model of diffusive relaxation and attenuation for liquid inclusions in a solid matrix. They apply this to attenuation in the earth's inner core. The present paper considers fluid media, including the earth's outer core. Analyses of the outer core by previous workers [Loper and Roberts, 1978; Gubbins, 1978; Anderson, 1980; Anderson and Given, 1982] are based entirely on the Vaisnys treatment.

In the acoustical literature, the most complete work on the attenuation of sound in suspensions and emulsions is by Epstein and Carhart [1953], and their theoretical formulation includes the irreversible entropy production associated with the localized temperature gradients near droplet surfaces. Their main application was to water fogs which, despite the large difference in density between gas phase and condensate, is somewhat analogous to the planetary environments considered here. Unfortunately, their formu- 
lation is not directly transferable to planetary problems, primarily because the frequencies of planetary interest $\left(10^{-6}-1 \mathrm{~Hz}\right.$, typically) are much less than the frequencies considered by acousticians. The theory developed here identifies a low-frequency dissipative regime which appears to have escaped attention previously, perhaps because it is not readily attainable in experiments. The remarkable feature of this new dissipative regime is that the quality factor $Q$, defined by

$$
Q=2 \pi\left|\frac{\text { energy stored }}{\text { energy dissipated/cycle }}\right|
$$

is a minimum at a frequency

$$
\omega_{0}=4 \pi D \bar{s} \eta
$$

which depends on the distance between droplets or snowflakes as well as their size. Here, $D$ is the solute diffusivity, $\bar{s}$ is an appropriately defined average inclusion radius, inclusions. Since the time to diffuse solute between inclusions can be very long, the 'resonant' frequency can be very low. For example, if we choose plausible numbers such as $D \sim 10^{-4} \mathrm{~cm}^{2} \mathrm{~s}^{-1}, \bar{s} \sim 10^{-3} \mathrm{~cm}$, and $\eta \sim 100$ $\mathrm{cm}^{-3}$, then $\omega_{0} \sim 10^{-4} \mathrm{~s}^{-1}$, which is within the range of frequencies of planetary interest. (The choice of $\eta$ and $\bar{s}$ in this example is not likely to be obvious to the reader at this stage but is discussed later.) In the water fogs considered by Epstein and Carhart [1953]. $\omega_{0} \sim 0.1 \mathrm{~s}^{-1}$, whereas the experiments were carried out at $\geq 10^{3} \mathrm{~Hz}$ and the regime of dissipation discussed in this paper was not encountered. The value of $Q$ at $\omega_{0}$ does not depend strongly on the details of the suspension particle distribution and can be as low as $-10^{2}-10^{3}$.

The analysis begins in section 2 with a consideration of the thermodynamic response to an oscillatory pressure wave of well-separated spherical particles suspended in a fluid phase. Thermal and compositional diffusive profiles are evaluated, and the resulting irreversible entropy production is determined. For this model, $Q \propto \omega^{1 / 2}$ in the high frequency limit and $Q \propto \omega$ in the low-frequency limit. The problem of a distribution of closely spaced suspended particles is analytically difficult and is approximated by first considering a regular lattice of particles and then generalizing the results to make approximate conclusions for suspensions of nonuniform particles, irregularly spaced. It is shown that the low-frequency behavior $Q \propto \omega$ for wellseparated particles is truncated at $\omega_{0}$ when particle interactions are considered, and $Q \propto \omega^{-1}$ for $\omega<\omega_{0}$.

In Saturn and possibly Jupiter, helium raindrops form because of the limited solubility of helium in metallic hydrogen [S/(')'enion, 1982a]. In section 3, the theory is applied to these helium 'rain clouds' and shown to be capable of providing the planetary tidal $Q-10^{5}$ that is required to explain the volcanism of the satellites Io and Enceladus. (The tidal heating and orbital evolution of satellites depends on the tidal $Q$ of both central planet and satellite. If the $Q$ of Saturn or Jupiter were much higher, then it would be difficult to explain the forced eccentricities of these satellites.)

In the earth's outer core, gradual cooling over geologic time may cause freeze-out of silicate particles (i.e., the core becomes silicate saturated) and solid iron particles. Appli- cation of the dissipation theory in section 4 indicates that significant damping of radial modes of free oscillation in the outer core is conceivable, as advocated by Anderson [1980].

The paper ends in section 5 with a critical assessment of the problems and difficulties with the model, most of which center on the large uncertainties in the existence and nature of the proposed suspensions. A brief comparison is made with the complementary work of Loper and Fearn [1982].

\section{DISSIPATION IN A SUSPENSION}

Consider an unperturbed state consisting of a homogeneous, isothermal fluid in which a single inclusion (droplet or snowflake) of a coexisting phase is placed at the origin, $r=0$. Let $T_{p h}\left(x^{\prime}, p^{\prime}\right)$ define the temperature at the phase boundary between the fluid and the inclusion. The pressure solute in the fluid is $x^{\prime}$, and the inclusion is assumed to consist of the pure solute. (This latter assumption can be relaxed without significantly altering the results provided the compositions of fluid and inclusion are substantially different.) Assume that the pressure undergoes a small oscillation about an unperturbed equilibrium state, so that the pressure, temperature, fluid composition, and inclusion radius can be expressed as

$$
\begin{aligned}
& p^{\prime}=p_{o}+p e^{i \omega t} \\
& T^{\prime}-T_{o}+\left[\left[\frac{\partial T}{\partial p}\right]_{\sigma, x} p+T(r)\right] e^{i \omega t} \\
& x^{\prime}=x_{o}+x(r) e^{i \omega t} \\
& s^{\prime}=s_{o}+s e^{i \omega t}
\end{aligned}
$$

where the primed quantities represent totals, the subscript zero refers to the unperturbed state, the unprimed quantities are perturbations, and $\omega$ is the driving frequency. The adiabatic response of the temperature field is assumed equal for the two phases and is explicitly included $(\sigma$ is the specific entropy), so that $T(r)$ is a localized temperature perturbation. The addition of the complex conjugate is implied for any measurable quantity; $p$ is taken to be real, but the other perturbation quantities will be complex in general.

It is assumed that $|s|$ is much smaller than any other length scale relevant to the problem (e.g., diffusion lengths). This will be justified a posteriori. It is assumed that the pressure perturbation does not cause the nucleation of new inclusions. (This point will be returned to at the end of the paper.) The diffusion equation defining the perturbation temperature field is then

$$
i \omega \theta_{ \pm}=K \frac{\partial^{2} \theta_{ \pm}}{\partial r^{2}}
$$

where $\theta \equiv r T(r), K$ is the thermal diffusivity of the medium (assumed equal for the phases), and,+- refer to $r>s_{o}, r<s_{o}$, respectively. The asymptotic and boundary conditions are 


$$
\begin{array}{cc}
\theta_{-}=0 & r=0 \\
\theta_{+}=\theta_{-} & r=s_{o} \\
\theta_{+} \rightarrow 0 & r \rightarrow \infty
\end{array}
$$

for which the appropriate solutions are

$$
\begin{gathered}
\theta_{-}=s_{o} T_{1} \sin k r / \sin k s_{o} \\
\theta_{+}=s_{o} T_{1} e^{-i k\left(r-s_{o}\right)} \\
k \equiv(1-i) k_{1} \\
k_{1} \equiv\left[\frac{\omega}{2 K}\right]^{1 / 2}
\end{gathered}
$$

where $T_{1}$ is still undetermined. Similarly, if $\chi \equiv r x(r)$ then the appropriate solution for the solute field is

$$
\begin{gathered}
\chi=s_{o} x_{1} e^{-i q\left(r-s_{0}\right)} \\
q \equiv(1-i) q_{1} \\
q_{\mathrm{l}} \equiv\left[\frac{\omega}{2 D}\right]^{1 / 2}
\end{gathered}
$$

where $x_{1}$ is still undetermined.

At $r=s_{o}$, the fluid must be in phase equilibrium with the inclusion:

$$
\left[\left\lceil\frac{\partial T_{p h}}{\partial p}\right]_{x}-\left[\frac{\partial T}{\partial p}\right]_{\sigma, x}\right] p+\left(\frac{\partial T_{p h}}{\partial x}\right]_{p} x=T \nmid=s
$$

In addition, the difference in heat fluxs between $r=s_{o}-\delta, r=s_{o}+\delta\left(\delta \ll s_{o}\right)$ must be supplied by the latent heat of material changing phase, and this source or sink of solute must determine the compositional gradient at the interface:

$$
\begin{gathered}
-\left.\rho C_{p} K \frac{\partial T}{\partial r}\right|_{r-s_{0}^{+}}=-\left.\rho C_{p} K \frac{\partial T}{\partial r}\right|_{r=s_{0}-}+\rho L i \omega s \\
-\left.D \frac{\partial x}{\partial r}\right|_{\left.\right|^{r-s_{o}}}=-i \omega s
\end{gathered}
$$

where $C_{p}$ is the specific heat, $\rho$ is the density and $L$ is the latent heat released per unit mass of inclusion formed. Equations (6)-(10) can be solved to yield

$$
\begin{gathered}
T_{1}=\left[\left[\partial T_{p h} / \partial p\right]_{x}-[\partial T / \partial p]_{\sigma, x}\right] p / A_{1} \\
x_{1}=\left[\left[\partial T_{p h} / \partial p\right]_{x}-[\partial T / \partial p]_{\sigma, x}\right] p / A_{2} \\
A_{1} \equiv 1-\left[\partial T_{p h} / \partial x\right]_{p}\left[k s_{o}\left(i+\cot k s_{o}\right) / \tau T_{L}\left(1+i q s_{o}\right)\right]
\end{gathered}
$$

$$
\begin{gathered}
A_{2} \equiv\left\langle\partial T_{p h} / \partial x\right]_{p}+\tau T_{L}\left(1+i g s_{o}\right) / k s_{o}\left(i+\cot k s_{o}\right) \\
\tau \equiv D / K \quad T_{L} \equiv L / C_{p}
\end{gathered}
$$

It is now straightforward to calculate the irreversible entropy production given by [Landau and Lifshitz, 1959, p. 225]

$$
\begin{gathered}
\frac{\partial}{\partial t} \int \rho \sigma d V= \\
\int \frac{\rho C_{p} K(\nabla T)^{2} d V}{T_{o}^{2}}+\int \rho D\left(\frac{\partial \mu}{\partial x}\right)_{T, p} \frac{(\nabla x)^{2}}{T} d V
\end{gathered}
$$

where $\sigma$ is specific entropy, $\mu$ is the solute chemical potential, and the integrals extend over all space. The time averages of the oscillatory functions $(\nabla T)^{2}$ and $(\nabla x)^{2}$ must be made on the right-hand side. However, it is first convenient to identify different limiting cases. Throughout the analysis, it is assumed $\tau<<1(D \ll K)$. In both giant planet and terrestrial cores; $D \sim 10^{-3}-10^{-4} \mathrm{~cm}^{2} / \mathrm{s}$, $K \sim 10^{-1} \mathrm{~cm}^{2} / \mathrm{s}$, and $\tau \sim 10^{-2}-10^{-3}$.

Case I: $q_{1} s_{o} \gg k_{1} s_{o} \gg 1$

In this limit, cot $k s_{o} \rightarrow 1$ and

$$
T_{1}=-\frac{1}{2}(1+i) \tau^{1 / 2} T_{L} p \frac{\left[\left[\partial T_{p h} / \partial p\right]_{x}-[\partial T / \partial p]_{\sigma, x}\right]}{\left[\partial T_{p h} / \partial x\right]_{p}}
$$

$$
x_{1}=-p \frac{\left[\left\lfloor\partial T_{p h} / \partial p\right]_{x}-[\partial T / \partial p]_{\sigma, x}\right]}{\left[\partial T_{p h} / \partial x\right]_{p}}
$$

Notice that the temperature perturbation is small; most of the effect of the pressure change is accommodated by a compositional boundary layer because of the low diffusivity of solute. The complete solution for the temperature field at $r>s_{o}$ is

$$
\begin{gathered}
T \cong 2\left|T_{1}\right| e^{-k_{1}\left(r-s_{0}\right)} \mid \cos \left(k_{1} \overline{r-s_{o}}-\omega t\right) \\
\left.+\sin \left(k_{1} \overline{r-s_{o}}-\omega t\right)\right]
\end{gathered}
$$

The contribution to the entropy integral in (13) comes equally from the two sides of the phase boundary and is given by

$$
\begin{gathered}
\int \rho C_{p} K\left(\frac{\nabla T}{T_{o}}\right)^{2} d V=8 \pi D\left[\frac{T_{*}}{T_{o}}\right]^{2}\left[\frac{p}{B}\right]^{2} \rho C_{p} k_{1} s_{o}^{2} \\
T_{*} \equiv T_{L} B \frac{\left[\left\lfloor\partial T_{p h} / \partial p\right]_{x}-[\partial T / \partial p]_{\sigma, x}\right]}{\left[\partial T_{p h} / \partial x\right]_{p}} \\
B \equiv \rho\left(\frac{\partial p}{\partial \rho}\right]_{\sigma}
\end{gathered}
$$


where $B$ is the isentropic bulk modulus. The contribution from the solute gradient to the entropy production is

$$
\begin{gathered}
\int \rho D\left(\frac{\partial \mu}{\partial x_{o}}\right]_{T, p} \frac{(\nabla x)^{2}}{T} d V=8 \pi D\left(\frac{x^{2}}{x_{o}}\right)\left[\frac{p}{B}\right]^{2} \rho \mathrm{R} q_{1} s_{o}^{2} \\
x_{*} \equiv B \frac{\left[\left\lfloor\partial T_{p h} /\left.\partial p\right|_{x}-\left[\partial T /\left.\partial p\right|_{\sigma, x}\right]\right.\right.}{\left(\partial T_{p h} / \partial x\right]_{p}}
\end{gathered}
$$

where $\mathbf{R}$ is the gas constant and the compositional dependence of $\mu$ is assumed to be dominated by the ideal mixing term $R T \ln x_{0}$. (This is not essential to the calculation, but neither is it likely to be wrong by a large factor. Most importantly, $\partial \mu / \partial x$ cannot be very small, except near absolute zero, because it is the entropy.)

Since $q_{1} \gg k_{1}$, a comparison of (16) and (17) indicates that the compositional term is larger by a factor of $\tau^{-1 / 2} \sim 10-100$ in planets. Differences in the other factors are generally smaller. The final estimate for the dissipation rate, expressed as the rate of increase of thermal energy per unit volume, $E_{t h}$, is obtained by multiplying the entropy production by $T$ and by the number density of suspended particles $\eta$ :

$$
\frac{d E_{t h}}{d t}=8 \pi D \mathrm{R} T\left[\frac{x^{2}}{x_{o}}\right]\left[\frac{p}{B}\right]^{2} \rho q_{1} s_{o}^{2} \eta
$$

This assumes that the particles are well enough separated that their associated diffusive effects do not overlap (see below). Since $q_{1} \propto \omega^{1 / 2}$, it is clear that the energy dissipated per cycle $\propto \omega^{-1 / 2}$ and the quality factor (equation (1)) $Q \propto \omega^{1 / 2}$ in this high-frequency limit. (We delay explicit evaluation of $Q$ until the various cases are examined.)

\section{Case II: $q_{1} s_{o} \gg 1 \gg k_{1} s_{o}$}

In this limit, the compositional gradients are still localized near the inclusion surface but the thermal gradients are extended. Since cot $k s_{o}=\left(i k s_{o}\right)^{-1}$ if $\left|k s_{o}\right| \ll 1$, it follows that $T_{1}$ is even smaller than previously (i.e., $T_{1} \propto \tau$ ). This is partially offset by the fact that $\nabla T \sim T_{1} / s_{o}$ rather than $k_{1} T_{1}$ because $T \sim r^{-1} e^{-i k\left(r-s_{0}\right)}$. Nevertheless, the compositional contribution to dissipation remains as before and larger by a factor $\sim \tau^{-1 / 2}$. This case is therefore no different from case $I$.

$$
\text { Case III: } 1 \gg q_{1} s_{o} \gg k_{1} s_{o}
$$

The contribution from thermal gradients is still small, but the compositional gradients are now changed:

$$
x=-2 x_{*}\left[\frac{p}{B}\right]\left[\frac{s_{o}}{r}\right] e^{-q_{1}^{\left(r-s_{o}\right)}} \cos \left(q_{1} \overline{r-s_{o}}-\omega t\right)
$$

and the resulting dissipation is now

$$
\frac{d E_{t h}}{d t}=4 \pi D R T\left(\frac{x^{2}}{x_{o}}\right)\left[\frac{p}{B}\right)^{2} \rho s_{o} \eta
$$

This is independent of frequency, implying $Q \propto \omega$.

At very low frequencies, the importance of this dissipation increases. How low in frequency can one go before the model is invalid? One possible limitation is implied by the assumption that changes in the size of the inclusion can be ignored in the diffusion equation. Mathematically, the criterion to be satisfied is

$$
\left|\frac{\partial s^{\prime}}{\partial t} \frac{\partial x^{\prime}}{\partial r}\right| \ll\left|\frac{\partial x^{\prime}}{\partial t}\right|
$$

Using (10), this can be expressed as

$$
q_{1} s_{o} \gg x_{1}
$$

This limitation is nonlinear; it depends on the magnitude of the pressure perturbation. It is unimportant in both the earth's core $\left(x_{1} \leq 10^{-9}\right.$; equation (20) is violated at $\omega \sim 10^{-16} \mathrm{~Hz}$ if $\left.s_{o} \geq 10^{-3} \mathrm{~cm}\right)$ and giant planets $\left(x_{1} \leqslant 10^{-8}\right.$; equation (20) is violated at $\omega-10^{-14} \mathrm{~Hz}$ if $s_{o} \geq 10^{-3} \mathrm{~cm}$ ). The estimates for $x_{1}$ are obtained from typical seismic and tidal pressure perturbations, respectively.

A more important limitation arises from the effect of other droplets in the suspension. If the diffusion distance $(D / \omega)^{1 / 2}$ is much greater than the distance between particles, then it is no longer possible to treat the particles independently. At low enough frequencies, the interparticle medium will be 'flooded' with the diffusive effects of many particles, and the solute gradients near the particle surfaces will be greatly diminished. It follows that $Q$ is likely to increase as $\omega \rightarrow 0$. The quantification of this expectation is difficult and will not be attempted here with rigor. A simplified model of a lattice of equivalent particles is solved rigorously and then used as a basis for generalized conclusions.

Let the suspension particles occupy the sites of a regular lattice defined by $\left\{\mathbf{r}_{j}\right\}$, where $j$ is an integer label. By Bloch's theorem (see any elementary solid state textbook, e.g., Harrison [1970]) the solute distribution $x(\mathbf{r})$ is invariant with respect to discrete lattice translations and can be expressed as

$$
x(\mathbf{r})=\sum_{\mathbf{G}} x_{\mathbf{G}} e^{i \mathbf{G} \cdot \mathbf{r}}
$$

where the set of discrete reciprocal lattice vectors $\{\mathbf{G}\}$ have the property that $\mathbf{G} \cdot \mathbf{r}_{j}-2 \pi N$, and $N$ is an integer (including zero). The diffusion equation to be solved can be written as

$$
\frac{\partial x}{\partial t}=D \nabla^{2} x+S \delta\left(\mathbf{r}-\mathbf{r}_{i}-s_{o} \hat{\mathbf{r}}\right) e^{i \omega t}
$$

where $\delta$ is the three-dimensional $\delta$ function and $S$ is a 'source strength' which must be chosen to conform with (8)-(10) and represents the solute source/sink at particle surfaces. In the limit of interest, $x=x_{1}$ at $\mathbf{r}=\mathbf{r}_{l}+s_{o} \hat{\mathbf{r}}$, where is given by (14).

Substitution of (21) into (22) yields

$$
\begin{aligned}
& i \omega x_{\mathbf{G}} V=-D G^{2} x_{\mathbf{G}} V \\
& +S e^{i \omega t} \sum_{j} e^{i \mathbf{G} \mathbf{r}_{j}} \int e^{i \mathbf{G} \cdot \hat{r} j_{0}} d^{3}\left(\mathbf{r}-\mathbf{r}_{\jmath}\right)
\end{aligned}
$$


which can be easily evaluated to give

$$
\begin{gathered}
x(\mathbf{r})=4 \pi s_{o}^{2} \eta S \underset{\mathbf{G}}{\sum_{(} \frac{\operatorname{sinc}\left(G s_{o}\right) e^{\iota \mathbf{G} \mathbf{r}}}{\left(i \omega+D G^{2}\right)}} \\
\operatorname{sinc}(y) \equiv \sin y / y
\end{gathered}
$$

In the high-frequency limit, many reciprocal lattice vectors contribute to the sum and the substitution

$$
\underset{\mathbf{G}}{\boldsymbol{\Sigma}} \rightarrow \frac{\nu}{(2 \pi)^{3}} \int d^{3} G
$$

can be made, where $v$ is the specific volume. The previous results can then be reproduced (with $S \equiv D x_{1} / s_{o}$ ). However, if the frequency is low enough, then the substitution of an integral for a sum is not valid because the $\mathbf{G}=0$ term in (24) contributes a large fraction of $x(\mathbf{r})$. Physically, this zero wave vector contribution corresponds to a uniform distribution in real space. (Interestingly, this 'special' treatment of $\mathbf{G}=0$ is mathematically analogous to the formulation of Bose condensation in the theory of superfluid helium.)

Clearly, the interparticle medium approaches saturation when the $\mathbf{G}=0$ contribution to (24) becomes larger than the summed contributions from $\mathbf{G} \neq 0$. The former is $4 \pi s_{o}^{2} \eta S / i \omega$, the latter is $\sim s_{0}^{2} S / D r\left(\sim s_{o} S / D\right.$ at $\left.r=s_{o}\right)$. Notice that these terms are out of phase by $\pi / 2$. The frequency at which these are comparable in magnitude is the 'resonant' frequency $\omega_{o}-4 \pi D s_{o} \eta$ of (2). Below this frequency, $S=i \omega x_{1} / 4 \pi s_{o}^{2} \eta$, and the solute concentration change between the surface of a particle and the interparticle medium is $\sim\left(\omega / \omega_{0}\right) x_{1}$. The irreversible entropy production is then as in case III above except that it is reduced by a factor $\sim\left(\omega / \omega_{o}\right)^{2}$. The contribution from thermal gradients is small and $Q \propto \omega^{-1}$.

The generalization to a lattice of nonuniform particle sizes is straightforward in the asymptotic limits. In the high-frequency limit, each particle contributes independently and the energy dissipated per unit volume is as in (19) except that $s_{o}$ is replaced by

$$
\int_{0}^{\infty} s_{o} \eta\left(s_{o}\right) d s_{o}
$$

where $\eta\left(s_{o}\right)$ is the number density of particles between radii $s_{o}, s_{o}+d s_{j}$. In the low-frequency limit, $S=i \omega x_{1} / 4 \pi \int s_{o}^{2} \eta\left(s_{o}\right) d s_{o}$ and

$$
\begin{aligned}
& \frac{d E_{t h}}{d t} \cong 4 \pi D \mathrm{R} T\left[\frac{x^{2}}{x}\right]\left(\frac{p}{B}\right)^{2} \rho \\
& \times\left\lfloor\frac{\omega}{4 \pi D}\right)^{2} \frac{\int s_{o}^{3} \eta\left(s_{o}\right) d s_{o}}{\left[\int s_{o}^{2} \eta\left(s_{o}\right) d s_{o}\right]^{2}}
\end{aligned}
$$

If we define $\omega_{o}$ as the frequency at which these two formulae (19) and (25) are equal then

$$
\omega_{o}=4 \pi D\left[\frac{\left[\int s_{o}^{2} \eta\left(s_{o}\right) d s_{o}\right]^{2} \int s_{o} \eta\left(s_{o}\right) d s_{o}}{\int s_{o}^{3} \eta\left(s_{o}\right) d s_{o}}\right]^{1 / 2}
$$

This defines the appropriate average $\bar{s}$ in (2).

If the particles are not arranged on a lattice, then the results can be formulated in terms of (unknown) pair distribution functions or structure factors, analogous to the formulation of the scattering properties of a liquid. However, the following simple heuristic argument indicates that the results are not fundamentally different from a lattice, provided the suspension properties are not 'pathological' (e.g., highly nonrandom clumping).

Near a given particle, the solute distribution is $-X_{n}\left(s_{o} / r\right)+X_{f}$ where the 'far' contribution $X_{f}$ is the diffusive effect of all the other particles within a diffusion distance $\sim(D / \omega)^{1 / 2}$ :

$$
X_{f} \simeq \eta \int_{0}^{(D / \omega)^{n}} 4 \pi r^{2} \cdot X_{n}\left[\frac{s_{o}}{r}\right) d r=4 \pi D \eta s_{o} X_{n} / \omega
$$

It follows that the 'far' contribution begins to dominate (i.e. $X_{f}>X_{n}$ ) for $\omega<\omega_{o}-4 \pi D \eta s_{o}$, defined as before. The particle distribution is unimportant because the diffusion distance $(D / \omega)^{1 / 2} \geq \eta^{-1 / 3}$, the interparticle separation.

The evaluation of $Q$ is straightforward for an acoustic (seismic) wave in the limit where the wavelength $\ll$ planetary radius. The energy stored in the pressure field is $4 p^{2} / B$ (the factor of 4 arising because the pressure field is $2 p \cos \omega t)$ and

$$
Q=\frac{4 p^{2} \omega}{B\left(d E_{t h} / d t\right)}
$$

where the dissipation is expressed per unit volume. At $\omega_{0}$, substitution of (19) into (27) gives

$$
Q\left(\omega_{o}\right) \equiv Q_{o}=4\left(\frac{B}{\rho \mathrm{R} T}\right) \frac{x_{o}}{x_{*}^{2}}
$$

This result is independent of diffusivity, particle size, and particle distribution! It depends only on thermodynamic parameters.

The evaluation of a tidal $Q$ is not quite as straightforward. Since the application of interest is giant planets, the formulation of Hubbard [1974] is followed. The dissipation must equal the work done on the satellite to change its orbit:

$$
\int \frac{d E_{t h}}{d t} \cdot d V=\frac{1}{2} M_{s} \omega T_{t}
$$

where the integral extends over the volume of the planet, $M_{s}$ is the satellite mass, $1 / 2 \omega$ is the angular velocity of the subsatellite point in the corotating frame, and $T_{t}$ is the tidal torque per unit mass

$$
T_{t}=\frac{9 \alpha G M_{s} a^{5}}{2 Q R^{6}}
$$

Here, $\alpha$ is a response coefficient $(\simeq 0.15$ for Jupiter and Saturn), $a$ is the planetary radius and $R$ is the distance to the satellite. In the particular case of an adiabatic $n=1$ polytrope discussed by Hubbard [1974], for which the pressure varies as the square of density, it can be shown that the tidal pressure perturbation is of order $\left(\omega^{2} a / g\right)_{\rho} V_{T}$, where $g$ is the gravitational acceleration and $V_{T}$ is the tidal 


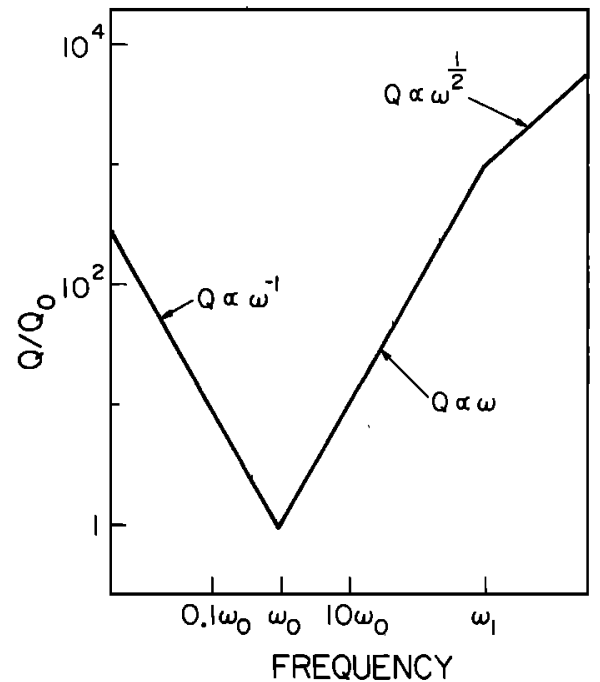

Fig. 1. Schematic representation of the frequency dependence of $Q$ (either seismic or tidal) for the suspension model, assuming a single particle size and uniform distribution. The frequency $\omega_{0}$ is defined by equation (2) and the (usually higher) frequency $\omega_{1}$ is $D \bar{s}^{-2}$.

potential (see the appendix). However, the region of helium droplet formation in the giant planets is stably stratified and the pressure perturbation is near $\rho V_{T}$ (see the appendix). It follows that

$$
\frac{p}{B}=\left\lfloor\frac{r}{a}\right\rfloor^{2} \cdot \frac{M_{s} a^{3}}{M_{p} R^{3}}
$$

where $r$ is a radial coordinate within the planet of mass $M_{p}$. A numerical coefficient somewhat different from unity is likely on the right-hand side, but its value is not known. The tidal $Q$ at $\omega=\omega_{0}$ obtained from (29) can be written as

$$
\left.\left.Q\left(\omega_{0}\right) \equiv Q_{o}=\beta\left[\frac{a}{\bar{r}}\right]^{4} \mid \frac{M_{p}}{M_{2}}\right] \int \frac{G M_{p}}{a \mathrm{R} T}\right]\left\lfloor\frac{x_{o}}{x^{2}}\right]
$$

where $\beta \simeq 0.2$ if the numerical coefficient in (31) is unity and $M_{2}$ is the total mass of the two-phase region centered about average radius $\bar{r}$. Specific numerical examples will be discussed in the next section, but typically $Q_{o}-10^{2}-10^{3}$.

In both seismic and tidal applications, the general formula for $Q(\omega)$ can be expressed as

$$
\begin{array}{rlrl}
Q(\omega) & =Q_{o}\left(\frac{\omega_{1}}{\omega_{o}}\right)\left[\frac{\omega}{\omega_{1}}\right]^{1 / 2} & & \omega \geq \omega_{1} \\
& \left.=Q_{o}\left(\frac{\omega}{\omega_{0}}\right]^{\frac{\omega_{o}}{\omega}}\right] & \omega_{o} \leq \omega \leq \omega_{1} \\
& =Q_{o} \leq \omega_{o}
\end{array}
$$

where $\omega_{1} \approx D \bar{s}^{-2}$. For broad particle distributions, the transitional frequencies will be poorly defined and the dependence of $Q$ on $\omega$ may be weaker. The minimum of $Q$ may occur over a decade or two of frequency, for example. Figure 1 shows the schematic $Q(\omega)$. The value of $Q_{0}$ will be considered in specific cases below, but for most con- ceivable circumstances it is greater than unity but not many orders of magnitude greater than unity.

These results can also be expressed in the form of an 'anomalous' bulk viscosity $\xi$ [Landau and Lifshitz, 1959, p. 304]. In the low-frequency limit,

$$
\xi=\frac{B}{\omega_{0} Q_{0}}
$$

For possible planetary values $\left(B-10^{12}\right.$ dynes $/ \mathrm{cm}^{2}$, $\left.\omega_{o} \sim 10^{-4} \mathrm{~s}^{-1}, Q_{o}-10^{2}\right) ; \xi-10^{14} \mathrm{P}$, which is typically about 16 orders of magnitude in excess of the molecular viscosity. At higher frequencies, the response of the medium cạn be represented by a complex bulk viscosity.

\section{Application to the Giant Planets}

It has long been suspected that the tidal $Q$ of Jupiter and Saturn is in the range $10^{5}-10^{7}$, such that significant tidal evolution of the orbits of inner satellites could take place in the age of the solar system [Goldreich and Soter, 1966]. It is now apparent from the observed tidally-driven volcanism of Io and the inferred tidal heating of Enceladus that the tidal $Q$ of Jupiter and Saturn are probably nearer the lower limit $\sim 10^{5}$ [Yoder and Peale, 1981; Yoder, 1981]. More precise quantification of the planetary $Q$ is not possible because of uncertainties in the primordial state, orbital histories and internal dissipative processes of the satellites. Several mechanisms have been proposed to provide a $Q$ approaching this lower limit: turbulent skin friction at an interface [Goldreich and Soter, 1966], excitation of inertial gravity waves in the atmosphere $(\mathrm{H}$. Houben and $\mathrm{P}$. Gierasch, unpublished, 1976), turbulent viscosity arising from thermal convection (Hubbard, 1976; but shown to be inadequate by Goldreich and Nicholson [1977]), and dissipation in the small, partial solid rock core [Dermott, 1979]. These are reviewed in detail by Yoder and Peale [1981]. Only Dermott's proposal comes close to satisfying the requirements, and his model is unfortunately based on very uncertain aspects of the interior models of Jupiter and Saturn (even more uncertain than the aspects about to be discussed)!

Using ideas very closely related to those developed in this paper, Stevenson [1980a] considered the irreversible entropy production at a global, horizontal phase transition interface between molecular and metallic hydrogen. This can provide a $Q$ of $10^{6}-10^{7}$, but it is difficult to approach $10^{5}$ unless there are resonances between the tidal perturbation and interfacial-inertial waves or normal modes of the planet. A more serious objection to this model concerns the nature of the molecular-metallic transition, which is probably not first order in pure hydrogen at $\sim 10^{4} \mathrm{~K}$, the relevant temperature. (It may, of course, be first order at $\sim 10^{3} \mathrm{~K}$; see Stevenson [1982a], for more details.)

In contrast, observational evidence for Saturn is strongly in favor of a first order phase transition in hydrogen-helium mixtures and a rain out of helium-rich droplets. The evidence is a helium depletion in the atmosphere [Hanel et al., 1981], a higher heat flow than would be expected simply from homogeneous cooling [Stevenson, 1980b; Hanel et al., 1982] and an almost exactly axisymmetric magnetic field [Connerney et al., 1982; Stevenson, 1982b]. Helium rain out is not a new idea; it was first proposed (for Jupiter) by 
Smoluchowski [1967] and in a substantially modified but more applicable form by Salpeter [1973]. The dynamics and application to Saturn followed later [Stevenson and Salpeter, 1977a,b; Stevenson, $1980 b$ ], and the reader is referred to Stevenson [1982a] for a review of current models. There is no observational evidence for helium rain out in Jupiter, but neither can it be excluded. Since Jupiter is hotter than Saturn, the insolubility of helium in hydrogen would be delayed to a later stage in the evolution (i.e., the last billion years, say), and the effect on atmospheric composition, heat flow, and magnetic field may yet be slight. In fact, the infrared observations allow a small depletion of helium from the Jovian atmosphere, relative to cosmic abundance [Gautier et al., 1981]. The existence of a twophase region in Jupiter must therefore be regarded as an open issue at present.

The value of $Q_{o}$ can be readily estimated for Jupiter and Saturn, using existing estimates for the solubility of helium [Stevenson, 1979a]. In the region of interest (total pressure -3 Mbar $) ;\left(\partial T_{p h} / \partial p\right)_{x}=-700 \mathrm{~K} / \mathrm{Mbar}, \quad(\partial T / \partial P)_{\sigma, x}$ $=850 \mathrm{~K} / \mathrm{Mbar},\left(\partial T_{p h} / \partial x\right)_{p}=4.8 \times 10^{4}, x_{o}=0.06$, and $x_{*}=0.2$. Applying equation (32) to Saturn; $(a / \bar{r})^{4}$ $\simeq 20, M_{p} / M_{2}=10, G M_{p} / a \mathrm{R} T=20, \beta-0.2$, and $Q_{0}$ $\simeq 10^{2}-10^{3}$. In Jupiter, $(a / \vec{r})^{4} \simeq 3$, but $M_{p} / M_{2}$ may be much larger and $Q_{o}-10^{2}-10^{3}$ again (but with much greater uncertainty on the upside).

The biggest uncertainty is in $\omega_{0}$. A detailed model of the population dynamics of helium droplets is not possible, if only because the large-scale fluid dynamics are very poorly known. There is one well-determined constraint, however, on the total flux of material undergoing differentiation (i.e., total 'rainfall'). Saturn has been undergoing differentiation for $\geq 2 \times 10^{9}$ years, during which time about $5 \%$ of the total mass of the planet has been transported as raindrops out of the two-phase region. The corresponding averaged mass flux $F$ is then about $4 \times 10^{-9} \mathrm{~g} \mathrm{~cm}^{-2} \mathrm{~s}^{-1}$. In Jupiter, the value could be comparable (since the total mass is much larger), or smaller. If $v(s)$ is the averaged drift velocity of droplets of radius $s$, then the flux constraint is

$$
F=\int \frac{4}{3} \pi \rho \eta(s) v(s) s^{3} d s
$$

(Here and subsequently, the subscript zero is omitted from $s_{o}$.) The simplest (but possibly incorrect) assumption to make is that the drift velocity is given by the usual Stokes formula or its turbulent equivalent [Landau and Lifshitz, 1959]:

$$
\begin{aligned}
v(s) & =5 \times 10^{4} s^{2} \quad \mathrm{~cm} / \mathrm{s} \quad\left(s<10^{-2} \mathrm{~cm}\right) \\
= & 60 \mathrm{~s}^{1 / 2} \quad \mathrm{~cm} / \mathrm{s} \quad\left(s>10^{-2} \mathrm{~cm}\right)
\end{aligned}
$$

where a kinematic viscosity of $4 \times 10^{-3} \mathrm{~cm}^{2} / \mathrm{s}$ has been assumed [Stevenson and Salpeter, 1977a]. If a single particle size is assumed, then each choice of $s$ defines $\eta$ by (35) and $\omega_{o}$ by (2):

$$
\begin{aligned}
& \omega_{o}=2 \times 10^{-16} \mathrm{~s}^{-4} \mathrm{~s}^{-1} \quad\left(s<10^{-2} \mathrm{~cm}\right) \\
& =2 \times 10^{-13} \mathrm{~s}^{-5 / 2} \mathrm{~s}^{-1}\left(s>10^{-2} \mathrm{~cm}\right)
\end{aligned}
$$

Equation (33) yields the tidal $Q$ (assuming

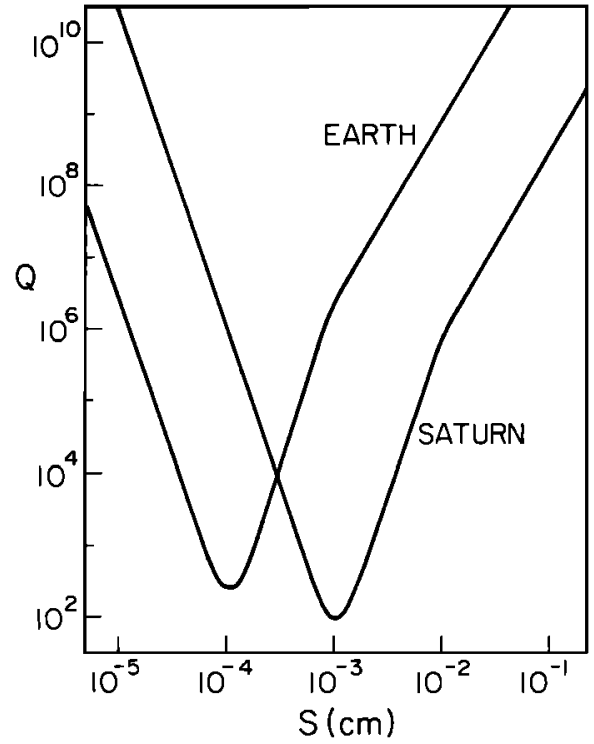

Fig. 2. Tidal $Q$ (Saturn) at $\omega=3 \times 10^{-4} \mathrm{~s}^{-1}$ and seismic $Q$ (earth) at $\omega=10^{-2} \mathrm{~s}^{-1}$, each as a function of particle radius, assuming the flux constraint (equation (35)). In reality, the twophase region is not likely to be homogeneous, and the flux constraint may only apply in an average sense. As a consequence, the very strong dependence on particle size may be an exaggeration. The minimal $Q-10^{2}$ is then likely to be an underestimate by one or two orders of magnitude.

$\omega=3 \times 10^{-4}$ ), shown in Figure 2 as a function of $s$. Since $Q$ is a very strong function of $s$, at least in this very simple example of a single particle size throughout the two-phase region, it is clear that some additional constraint must be found if $Q$ is to be estimated.

The particle size distribution depends on many poorly known or unknown factors: existence of nucleation sites, coagulation and fragmentation, droplet-droplet interactions, and fluid circulations in clouds-indeed, all the complexities which make terrestrial cloud physics so rich in phenomenology [e.g., Byers, 1965]. However, there are three rather different plausibility arguments which constrain the particle size; two of which coincidentally give $s-10^{-2}-10^{-3} \mathrm{~cm}$ and $Q-10^{s}$.

Consider, first, the hypothesis that a small, equilibrium, average supersaturation $\delta$ prevails throughout the two-phase medium, caused by secular cooling of the planet. Here, $\delta$ is defined as the fractional extent to which the actual fluid composition differs from the saturation value. Consider a control volume that is a few times larger than a typical droplet. The time $\tau$ between arrivals of a droplet into this control volume is then $\tau=\left(\eta v s^{2}\right)^{-1}$. During successive arrivals, the supersaturation $\delta$ builds up to a value $\sim \tau / \tau_{e v}$ where $\tau_{e v} \sim 10^{9}$ years (the time for $\sim 100 \%$ supersaturation to occur). During each droplet transit, the supersaturation is reset almost to zero since the droplet can grow by diffusion at the expense of the excess solute within the control volume. It follows that $\eta v s^{2} \delta \sim 10^{-17}$. Furthermore, the droplet continues to grow as $s=(\delta D t)^{1 / 2}$, where $t$ is the time elapsed since its initial nucleation. Using equation (36), assuming the viscous dominated (small $s$ ) limit, the total drift distance covered in time $T$ is $-25 \delta T^{2}$. Equating this to $5 \times 10^{8} \mathrm{~cm}$, a likely thickness for the twophase region [Stevenson, $1980 \mathrm{~b}$ ] gives a final droplet size $s=2 \delta^{1 / 4}$. Substituting above for $\delta$ gives 


$$
\eta v s^{6}=10^{-18}
$$

But the flux constraint gives

$$
\eta v s^{3}=10^{-9}
$$

from which it follows that $s-10^{-3} \mathrm{~cm}$. Of course, this is only an approximate calculation, and the resulting uncertainty in $Q$ is still two or three orders of magnitude.

An alternative argument supposes that droplets must grow to a size such that they can successfully compete with small-scale convection in their local environment (i.e., $\left.v_{\text {stokes }} \geq v_{\text {conv }}\right)$. The physical motivation for this assumption is that if $s$ were smaller then particles would be convectively transported into regions where they preferentially dissolve rather than settle out by gravity. A likely convective velocity [Stevenson, 1979 $\mathrm{b}$ ] is $\sim 1 \mathrm{~cm} / \mathrm{s}$ and $v_{\text {stokes }}-1 \mathrm{~cm} / \mathrm{s}$ for $s=5 \times 10^{-3} \mathrm{~cm}$. This approach is questionable because of large uncertainties in the convective velocities. Indeed, the two-phase region is expected to be relatively stable.

A third argument yields a different result. Suppose that there are no nucleating sites and the formation of droplets depends on homogeneous nucleation. The supersaturation is then large; droplets nucleate infrequently but then grow rapidly and fragment because the hydrodynamic drag on the droplet surface exceeds the restoring force of surface tension. This regime is discussed by Stevenson and Salpeter $[1977 b]$ and leads to droplets of typically $0.1-1 \mathrm{~cm}$ in radius. At first sight, this case would suggest a very low $\omega_{o}$ and very high $Q$. However, this regime is characterized by intermittent rainstorms [Salpeter and Stevenson, 1976] and the flux constraint (equation (35)) can no longer be used except in a time average. It is likely that these rainstorms are highly dissipative for short periods. Further attempted quantification would be highly speculative.

In summary, $s \sim 10^{-2}-10^{-3} \mathrm{~cm}$ and $Q \sim 10^{3}-10^{6}$ are possible, although by no means definitely established. The nature of the model prevents more precise quantification, but this should not be regarded as an argument against its underlying validity. The most striking feature of the model is that it is more than capable of providing the necessary dissipation. The two-phase region in Saturn or Jupiter is not likely to be spatially homogeneous and only part needs to be at or near minimal $Q$.

\section{Application to TerRestrial Fluid CORES}

The fundamental radial free oscillation mode ${ }_{o} S_{o}$ for the earth has an associated $Q=5000$ [Anderson, 1980]. The physical interpretation of this observation is not unique and may not even involve the outer core, but one possible explanation attributes the dissipation to the fluid region of the core. If the losses occur only in this region then a local $Q-2 \times 10^{3}$ is required. If this were attributed to a bulk viscosity $\xi$, then in the low-frequency limit,

$$
Q_{K}^{-1}=\frac{\omega \xi}{\rho c^{2}}
$$

[Anderson, 1980 equation (1) which for $\rho=11.3 \mathrm{~g} \mathrm{~cm}^{-3}$, $c=9.6 \times 10^{5} \mathrm{~cm} \mathrm{~s}^{-1}$ and $\omega=6 \times 10^{-3}$ implies $\xi=8$ $\times 10^{11} \mathrm{P}$. (Anderson quotes $5 \times 10^{2} \mathrm{P}$, evidently a numerical error.) There is no plausible microscopic process in a fluid that could provide this viscosity. Indeed, the shear viscosity of the outer core is likely to be very similar to the low pressure value $\sim 10^{-2} \mathrm{P}$ [Stevenson, 1981] and the atomic bulk viscosity is not likely to be many orders of magnitude greater. The occasionally expressed view that viscosity increases as pressure increases may be true along an isotherm or even an adiabat but is not generally true for simple fluids if the ratio of actual temperature to melting temperature is kept constant. Indeed, computer simulations [Ashurst and Hoover, 1975; Hoover et al., 1980] support scaling laws of this sort, in which explicit dependence of viscosity on pressure is absent.

There are at least two possible sources of suspended particles which could provide substantial attenuation in the outer core. One involves the formation of solid, almost pure iron particles which settle to cause growth of the inner core [Loper and Roberts, 1978, 1980]. Another involves progressive freeze-out of silicate or magnesium oxide particles which rise to the core-mantle boundary [Braginsky, 1963; Schloessin and Jacobs, 1980]. The latter is not frequently discussed but is an inevitable consequence of any model in which the earth's core starts out hot and silicate saturated, assuming that the solubility decreases as the temperature decreases. In both cases, the two-phase region could be either thin or extended, depending on poorly known aspects of the phase diagram.

Likely parameter values are $B / \rho \mathrm{R} T \sim 80$ and $x_{0} / x_{*}^{2} \sim 1$ so that $Q_{o}$ (equation $(28)$ ) is $\sim 300$. As in the giant planet calculation, a flux constraint can be imposed, assuming that a few percent of the outer core have frozen out in the age of the solar system. The result is $F=3 \times 10^{-9} \mathrm{~g} \mathrm{~cm}^{-2} \mathrm{~s}^{-1}$ at the inner core-outer core boundary and $4 \times 10^{-10} \mathrm{~g} \mathrm{~cm}^{-2} \mathrm{~s}^{-1}$ at the core-mantle boundary. The drift velocity of particles is given by

$$
\begin{aligned}
v & =10^{4} \mathrm{~s}^{2} \mathrm{~cm} / \mathrm{s} \quad(\mathrm{s} \leq 0.02 \mathrm{~cm}) \\
& =30 \mathrm{~s}^{1 / 2} \mathrm{~cm} / \mathrm{s} \quad(\mathrm{s} \geq 0.02 \mathrm{~cm})
\end{aligned}
$$

where a kinematic viscosity of $10^{-2} \mathrm{~cm}^{2} / \mathrm{s}$ is assumed [Stevenson, 1981]. Assuming a diffusivity $D=10^{-4} \mathrm{~cm}^{2} / \mathrm{s}$, the 'resonant' frequency $\omega_{0}$ can then be evaluated. The resulting $Q$ at $\omega=10^{-2} \mathrm{~s}^{-1}$ is shown as a function of $s$ in Figure 2. The dissipation is important (i.e., $Q \leq 10^{4}$ ) for $10^{-4.5} \leq s \leq 10^{-3.5} \mathrm{~cm}$ (although a more realistic model involving a size distribution would probably give a broader minimum in $Q$ ). For the particular example of $s=10^{-4} \mathrm{~cm}, Q(\omega)$ is shown in Figure 3 and compared with the model suggested by Anderson and Given [1982] from their analysis of the normal mode data. This is not, of course, a confirmation of the model (which fortuitously has a similar functional form) but merely a demonstration of compatibility.

There is no compelling argument for a particle size $\sim 10^{-4} \mathrm{~cm}$ (one micron). The equilibrium supersaturation' argument discussed in the previous section suggests $s \sim 10^{-2} \mathrm{~cm}$. Convective velocities suggested by geomagnetic westward drift are $\sim 10^{-2} \mathrm{~cm} / \mathrm{s}$ and Stokes velocities are comparable for $s=10^{-3} \mathrm{~cm}$. If the particles are solid rather than liquid, then there is no upper limit to particle size. If liquid droplets occur, then hydrodynamic breakup limits the droplet size to $s \leq 0.1 \mathrm{~cm}$. The total mass 
fraction of the medium in the form of suspended particles can be very small even when the dissipation is large: for $s=10^{-4} \mathrm{~cm}, \eta=10^{5} \mathrm{~cm}^{-3}$, and $-10^{-6}$ of the mass is involved. It is conceivable that some regions have very high dissipation, whereas other regions of the core have very low dissipation. This will, however, increase the core average $Q$ and make it less likely that the model of Figure 3 is achievable by this mechanism. This model also suggests significant dissipation of earth tides $\left(\omega-10^{-4} \mathrm{~s}^{-1}\right)$ is possible, especially if the particle size is typically somewhat larger (e.g., $10^{-3} \mathrm{~cm}$ ).

In summary, the suspension model provides a conceivable mechanism for the damping of radial modes or lower frequency perturbations (e.g., tides) in the earth, but it must be regarded as a marginal explanation at best because the 'resonant' frequency needs to be near the actual mode frequency. No strong argument can be advanced for such a coincidence. This criticism is not as applicable to the giant planets where a $Q \sim 10^{5}$ can be achieved as the average of a spatially varying environment, only small parts of which need to be at or near resonance.

\section{CONCLUDING COMMENTS}

A model has been presented which demonstrates that a dilute suspension of one phase as droplets or snowflakes in another (compositionally different) phase can be highly dissipative. In the limit of low-frequency and small pressure perturbations, the medium behaves as though it has a bulk viscosity of (typically) $-10^{12} \mathrm{P}$. No attempt has been made to quantify the model with high precision because the necessary parameters (particle size and number density) are so poorly constrained. Nevertheless, plausible parameters lead to substantial dissipation in planetary cores for tidal or seismic perturbations.

There has also been no attempt to treat the problem of larger pressure perturbations, for which nucleation of new droplets may occur at pressure peaks and an asymmetric response occurs, rendering the diffusion problem nonlinear. For the perturbations of interest $\left(|p| / B \sim 10^{-7}\right.$ to $\left.10^{-9}\right)$, this is unlikely to be important, even though the instantaneous supersaturation may thereby be larger than the 'equilibrium' $\delta$ discussed above. The reason is that most nucleated droplets must survive and the creation rate (number $/ \mathrm{cm}^{3} / \mathrm{s}$ ) is therefore $\sim \eta v / d$, where $d$ is the thickness of the two-phase region. For the examples discussed, the time between creation events in a specified control volume is many orders of magnitude greater than the time between arrivals (by drift) of a droplet into the control volume.

Loper and Fearn [1982] have analyzed the problem of pressure oscillations in a medium consisting of melt inclusions in a solid matrix. The major difference between their analysis and the present work is that they could ignore the diffusion of atoms in the solid, whereas solute diffusion in the liquid between solid inclusions is crucial to the present analysis. This difference leads to the possibility in their analysis of two minima in $Q(\omega)$ at frequencies substantially different from the 'resonant' frequency $\omega_{o}$ discussed here.

Finally, it is worth noting that since two-phase regions evolve on a geologic time scale, the tidal dissipation factor $Q$ must also evolve. This observation is almost trivial but deserves emphasis because of the frequency with which

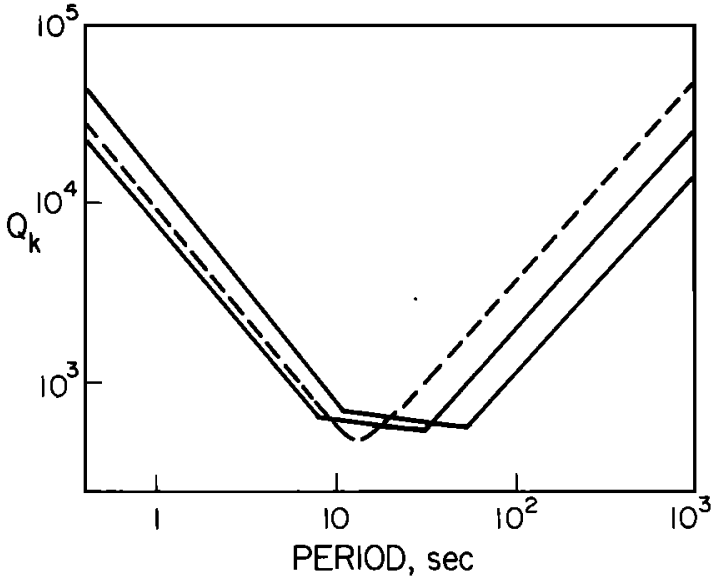

Fig. 3. Seismic $Q$ as a function of period for the earth's outer core. The dashed line is for the theoretical model assuming $\omega_{o}-10^{-2} \mathrm{~s}^{-1}$. The two solid lines are models obtained by Anderson and Given [1982] for two layers in the outer core. The similarity does not imply support for the model, merely compatibility.

papers appear in which authors assume that the $Q$ of some body is constant. This is almost never a reasonable assumption for most mechanisms (not just the mechanism discussed here). If the present tidal $Q$ of Jupiter is caused by the geologically recent formation of a two-phase region, then Io's volcanism (and perhaps even the orbital commensurabilities of the Galilean satellites) might also be geologically recent.

\section{APPENDIX: TIDAL PRESSURE PULSES}

For a perturbing tidal potential which varies as $e^{i \omega t}$ in the rotating frame of the planet, the pressure perturbation $p$ introduced equation (3) must be obtained from the 'convective' derivative

$$
p=\frac{1}{i \omega}\left[\frac{\partial p_{T}}{\partial t}+(\overrightarrow{\mathbf{u}} \cdot \nabla) p_{o}\right]
$$

where $p_{o}$ is the equilibrium pressure field, $p_{T}$ is the tidal perturbation and $\overrightarrow{\mathbf{u}}$ is the tidal flow field. Hubbard [1974] analyzed an adiabatic $n=1$ polytrope for which $p^{\prime}=K \boldsymbol{\rho}^{\prime 2}$, where $K$ is a constant and the primed quantities are total pressure and density. The $n=1$ polytrope is a good approximation to the giant planets, at least in the absence of phase transitions. However, he considered only equilibrium tidal perturbations for the density and a potential tidal flow field. This is not dynamically consistent, as Houben [1978, and private communication, 1982] has pointed out. The correct procedure, is as follows.

Let $\rho^{\prime}=\rho_{o}+\rho_{T}$ where $\rho_{T}=\rho_{T, e}+\rho_{2}, \rho_{T}$ is the total tidal density perturbation, $\rho_{T, e}$ is the equilibrium tidal response and $\rho_{2}$ is the (smaller) dynamic response. It is simple to show that $\rho_{T, e}$ is determined by

$$
\begin{gathered}
\rho_{T, e}=\phi / 2 K \\
\phi=V_{T}+V_{I, e} \\
V_{l, e}=-G \int \frac{\rho_{T, e}\left(\vec{r}^{\prime}\right) d^{3} \vec{r}}{\left|\vec{r}-\vec{r}^{\prime}\right|}
\end{gathered}
$$


where $V_{T}$ is the tidal potential provided by the external perturbing body. Clearly, the equilibrium radial displacement of an equipotential surface at any time or position is $\delta=-\phi / g$, where $g$ is the local gravitational potential. Since the time-varying part of the tidal potential has zero average on a sphere, the perturbed equipotential surface has the same volume (to lowest order) as the unperturbed equipotential surface and $\delta$ is also the displacement of a fluid element. The equilibrium contribution to $p$ in (A1) is then $2 K \rho_{o} \rho_{T, e}+\delta \rho_{o} g$, which is zero by (A2). The convective pressure perturbation depends entirely on nonequilibrium tidal effects as follows. If $\rho_{2} \ll \rho_{T, e}$, then the continuity equation is

$$
\frac{\partial \rho_{T, e}}{\partial t}+\nabla \cdot\left(\rho_{o} \mathrm{u}\right)=0
$$

which implies $|\mathbf{u}|-(a \omega) \rho_{T, e} / \rho_{o}$. The inviscid linearized Navier-Stokes equation is

$$
\begin{gathered}
\left.\frac{\partial \mathrm{u}}{\partial t}+2 \boldsymbol{\Omega} \times \mathrm{u}=\nabla \mid V_{I, 2}-2 K \rho_{2}\right) \\
V_{I, 2} \equiv-G \int \frac{\rho_{2}(\overrightarrow{\mathbf{r}}) \mathrm{d}^{3} \mathbf{r}^{\prime}}{\left|\overrightarrow{\mathbf{r}}-\overrightarrow{\mathbf{r}}^{\prime}\right|}
\end{gathered}
$$

from which it follows that $\rho_{2} \sim$ $(\omega a / K) \cdot|\mathbf{u}|-(\omega a / c)^{2} \rho_{T, e}$, where $c$ is the sound speed. As a consequence, $p=2 K \rho_{o} \rho_{2} \sim\left(\omega^{2} a / g\right) \rho_{o} V_{T}$.

However, the region in which helium-rich raindrops form is necessarily stably stratified because of a helium gradient [Stevenson and Salpeter, 1977b]. In this case, the 'equilibrium' contribution to the convective derivative (A1) is no longer zero, essentially because the tidal perturbations do not obey the same $p-\rho$ relationship as the static planet. (Of course, this also means that the terminology "equilibrium" can be misleading.) If an element of fluid is displaced through a distance $\delta$ then the density perturbation is $\Delta \rho-\left[(\partial \rho / \partial p)_{\sigma}-d \rho_{o} / d p_{o}\right] \rho_{o} g \delta$, where the second derivative refers to the actual (stable and nonadiabatic) density structure of the planet. In general ${ }_{\mid} d \rho_{o} / d p_{o} \mid \gg[\partial \rho / \partial p]_{\sigma}$ in the stable region. Since $\Delta \rho g-|\nabla \phi|$, it follows that the perturbation pressure is of order $\rho V_{T}$ and therefore much larger than the adiabatic case. The estimate of equation (31) follows, except for a numerical coefficient that can only be obtained by a detailed numerical analysis of the tidal response: This analysis has not been performed.

Acknowledgments. I wish to especially thank $\mathrm{H}$. Houben (NASA Ames) for his careful review of the manuscript, resulting in a complete rewriting of the appendix. This work is supported by NASA grant NAGW-185 and NSF grant EAR-8206383 and is contribution 3800 from the Division of Geological and Planetary Sciences, California Institute of Technology, Pasadena, California 91125 .

\section{REFERENCES}

Anderson, D. L., Bulk attenuation of the earth and viscosity of the core, Nature, 285, 204-207, 1980.

Anderson, D. L., and J. W. Given, Absorption band $Q$ model for the earth, J. Geophys. Res., 87, 3893-3904, 1982.

Ashurst, W. T. and W. G. Hoover, Dense fluid shear viscosity via nonequilibrium molecular dynamos, Phys. Rev., IIA, 658-678, 1975.
Braginsky, S. I., Structure of the $F$ layer and reasons for convection in the earth's core, Dokl. Akad. Nauk SSSR, 149, 8-10, 1963.

Byers, H. R., Elements of Cloud Physics, 191 pp., University of Chicago Press, Chicago, 1965.

Connerney, J. E. P., N. F. Ness, and M. A. Acuna, Zonal harmonic model of Saturn's magnetic field from Voyager 1 and 2 observations, Nature, 298, 44-46, 1982.

Dermott, S. F., Tidal dissipation in the solid cores of the major planets, Icarus, 37, 310-321, 1979.

Epstein, P. S. and R. R. Carhart, The absorption of sound in suspensions and emulsions, I, Water fog in air, J. Acoust. Soc. Am., 25, 553-565, 1953.

Gautier, D., B. Conrath, M. Flasar, R. Hanel, V. Kunde, A. Chedin, and N. Scott. The helium abundance of Jupiter from Voyager, J. Geophys. Res., 86, 8713-8720, 1981.

Goldreich, P. and P. D. Nicholson, Turbulent viscosity and Jupiter's tidal Q, Icarus, 30, 301-304, 1977.

Goldreich, $\mathrm{P}$. and S. Soter, $\mathrm{Q}$ in the solar system, Icarus, 5, 375-389, 1966.

Gubbins, D., Attenuation of seismic waves in an iron slurry, Geophys. Astrophys. Fluid Dyn., 9, 323-326, 1978.

Hanel, R. et al., Infrared observations of the Saturnian system from Voyager 1, Science, 212, 192-200, 1981.

Hanel, R., B. J. Conrath, V. G. Kunde, J. C. Pearl and J. A. Pirraglia, Albedo, internal heat flux and energy balance of Saturn, Icarus, in press, 1982.

Harrison, W. A. Solid State Theory, 554 pp., McGraw-Hill, 1970.

Hoover, W. G., A. J. C. Ladd, R. B. Hickman and B. L. Holian, Bulk viscosity via non-equilibrium and equilibrium molecular dynamics, Phys. Rev., 2lA, 1756-1760, 1980.

Houben, H., Tidal dissipation in the solar system and the possibility of tidally-driven planetary magnetic dynamics, $\mathrm{Ph} . \mathrm{D}$. thesis, 140 pp., Cornell Univ., Ithaca, N.Y., 1978.

Hubbard, W. B., Tides in the giant planets, Icarus, 23, 42-50, 1974.

Kieffer, S. W., Sound speed in liquid-gas mixtures: Water-air and water-steam, J. Geophys. Res., 82, 2895-2904, 1977.

Landau, L. D. and E. M. Lifshitz. Fluid Mechanics, 536 pp., Pergamon, London, 1959.

Litovitz, T. A. and C. M. Davis, Structural and shear relaxation in liquids, in Physical Acoustics, vol. IIA, edited by W. P. Mason and R. N. Thurston, pp. 281-349, Academic, New York, 1965.

Loper, D. E. and D. R. Fearn, A seismic model of a partially molten inner core, J. Geophys. Res., in press, 1982.

Loper, D. E. and P. H. Roberts, On the motion of an iron-alloy core containing a slurry. I. General Theory, Geophys. Astrophys. Fluid Dyn., 9, 289-321, 1978.

Loper, D. E. and P. H. Roberts, On the motion of an iron-alloy core containing a slurry. II. A simple model, Geophys. Astrophys. Fluid Dyn., 16, 83-127, 1980.

Salpeter, E. E., On convection and gravitational layering in Jupiter and in stars of low mass, Astrophys. J. Lett., 181, 83-86, 1973.

Salpeter, E. E. and D. J. Stevenson, Heat transport in a stratified two-phase fluid, Phys. Fluids, 19, 502-509, 1976.

Schloessin, H. H. and J. Jacobs, Dynamics of a fluid core with inward growing boundaries, Can. J. Earth Sci., 17, 72-89, 1980.

Smoluchowski, R., Internal structure and energy emission of Jupiter, Nature, 215, 691-695, 1967.

Stevenson, D. J., Solubility of helium in metallic hydrogen, $J$. Phys. F, 9, 791-800, 1979a.

Stevenson, D. J., Turbulent thermal convection in the presence of rotation and a magnetic field: A Heuristic theory, Geophys. Astrophys. Fluid Dyn., 12, 139-169, $1979 b$.

Stevenson, D. J., Dissipative processes and tidal $\mathrm{Q}$ for the major planets, Bull. Amer. Astron. Soc., 12, 696, 1980a.

Stevenson, D. J., Satum's luminosity and magnetism, Science, 208, 746-748, $1980 b$.

Stevenson, D. J., Models of the earth's core, Science, 214, 611-619, 1981.

Stevenson, D. J., Interiors of the giant planets, Ann. Rev. Earth Planet. Sci., 10, 257-295, $1982 a$.

Stevenson, D. J., Reducing the non-axisymmetry of a planetary dynamo and an application to Saturn, Geophys. Astrophys. Fluid Dyn., 21, 113-127, 1982b.

Stevenson, D. J. and E. E. Salpeter, The phase diagram and transport properties for hydrogen-helium fluid planets, Astrophys. $J$. Suppl., 35, 221-237, $1977 a$. 
Stevenson, D. J. and E. E. Salpeter, The dynamics and helium distribution in hydrogen-helium fluid planets, Astrophys. J. Suppl., $35,239-261,1977 b$.

Vaisnys, J. R., Propagation of acoustic waves through a system undergoing phase transitions, J. Geophys. Res., 73, 7675-7683, 1968.
Yoder, C. F., Tidal friction and Enceladus' anomalous surface, Eos Trans. $A G U, 62,939,1981$.

Yoder, C. F. and Peale, S. J., The tides of Io, Icarus, 47, 1-35, 1981.

(Received July 12, 1982; accepted October 15, 1982.) 